\title{
Radical surgery in oncology: a vision of the future
}

Received: 25 August 2004 / Accepted: 16 December 2004/Published online: 4 February 2005

(C) Springer-Verlag Berlin / Heidelberg 2005

To foresee the future, one has to look back to the past. Comparing what we do today to what we did 20 years ago may help in visualizing what we may do in 2024. My expectation is that surgery, especially radical surgery, will continue to play a lesser role and that the surgical procedures performed will be less radical. The reasons for this include less advanced disease due to early detection and prevention, increasing use of multimodality therapy, new technologies, patient expectations of less invasive treatment, and cost/benefit aspects of care.

Focusing on gynecological oncology, including breast cancer, in the past points the way to the future. Let's quickly review treatment trends for each major disease site.

\section{Cervical cancer}

Early detection and prevention, probably enhanced by human papilloma virus vaccination, will further decrease the rate of invasive and, especially, advanced invasive cancer of the cervix. The trend towards less radical surgery, including minimally invasive techniques, will continue, possibly enhanced by sophisticated operative robotics. This trend, combined with multimodality therapy combining radiation-chemo-immunotherapy and new targeted molecular treatment options, will further reduce the need for radical and ultraradical surgery in the primary as well as the recurrent setting. Sophisticated diagnostic tools such as magnetic resonance imaging and positron emission tomography/ computed tomography will further eliminate unnecessary surgical interventions for treatment planning and outcome monitoring. These trends will apply to cancers of the vulva and vagina in a similar fashion.

B.-U. Sevin · D. Wallwiener $(\bowtie)$

University Tübingen, Tübingen, Germany

E-mail: diethelm.wallwiener@med.uni-tuebingen.de

\section{Endometrial cancer}

The incidence of endometrial carcinoma will also continue to decrease. The declining use of estrogen replacement therapy and the increasing awareness and better management of risk factors such as obesity and diabetes mellitus will further decrease the incidence of uterine cancer and increase early detection rates. Hysterectomy by laparotomy or laparoscopy, with or without lymph node dissection, in combination with adjuvant therapy will continue to further increase cure rates and reduce the need to treat recurrent cancer. Improved adjuvant combination therapies combined with better post-treatment surveillance after primary treatment will make surgery less frequently necessary as well as the less likely treatment of choice for recurrent disease.

\section{Ovarian cancer}

Although early detection and prevention of ovarian cancer is still an unfulfilled hope, proteinomics and genomics may in the near future make early detection a realistic and affordable reality. Together with prophylactic oophorectomy for the small but significant subset of women with identifiable risk (classification of whom is expected to become more sophisticated with time), the incidence of ovarian cancer is expected to decline. While tumor debulking in combination with chemotherapy is currently the standard of care, requiring extraordinary surgical skill to be optimally performed, it can be expected that with better and bettertolerated systemic therapy, the need for radical surgery will decline. New and improved adjuvant combination therapy, including treatment of individual molecular tumor targets, will improve treatment outcomes and may further reduce - or even eliminate - the need for radical surgery. This will hold true for both primary and recurrent disease. Interventional radiology will play an 
increasing role in managing patients with complications, such as small bowel obstruction, especially in the terminal disease state.

\section{Breast cancer}

In no other area of female cancer care has the change from radical surgery to multimodal combination therapy been more obvious and conceptionally convincing than in breast cancer. Radical mastectomy has been replaced by wide tumor resection and minimally invasive sentinel node biopsy, combined with adjuvant radiation/chemotherapy/hormone therapy. Add to that trend the expected but still unimaginable contributions of molecular biology and evolving new treatment options, and it becomes obvious that radical surgery will not be playing a major role in the future.

Standards of care will also change dramatically for other reasons, such as patient expectations/demands, treatment side effects, and cost-benefit data in a world of increasing health care costs. None of these will favor expansion of radical and ultraradical surgery.

In this new world of medicine, the challenge will be to prepare future gynecological cancer surgeons to be able to acquire and maintain those skills required to optimally perform the surgical procedure that will remain a shrinking but integral part in the armamentarium of comprehensive multimodality care of women with gynecological malignancies. 